\title{
Wandering eye for RNAi
}

New findings call into question the premise behind prominent clinical trials testing small interfering RNA (siRNA) against age-related macular degeneration, a leading cause of blindness. In these trials, researchers aim to ease symptoms by knocking out expression of vascular endothelial growth factor (VEGF) or its receptor, which contribute to growth of excess blood vessels in this disease. But experiments in mice ${ }^{1}$ suggest that the method does not work through RNA interference (RNAi); instead it works through activation of Toll-like receptor 3 and induction of components of the innate immune response.

\section{John Rossi:}

Age-related macular degeneration seemed ideal for clinical trials, in part because naked, unmodified siRNAs can be injected directly into the confined space of ocular tissue. Even though there was little knowledge of how the siRNAs entered the target cells, clinical trials were initiated on the assumption that RNAi mediated the inhibition of vascularization in the eye in animal models.

It was therefore surprising, if not shocking, to read the new study. Among the take-home messages are the importance of appropriate controls in preclinical studies and of the use of delivery strategies that shield siRNAs from the innate immune receptors. The authors optimistically suggest that activation of TLR3 by siRNA can be harnessed to treat various diseases, but, realistically, the findings are a setback for RNAi therapeutic applications in which sequence-specific target knockdown is the primary goal.

Professor, Division of Molecular Biology, Beckman Research Institute of the City of Hope, Duarte, California, USA.

\section{Phil Zamore:}

These data raise concerns about siRNAs delivered directly to mammalian cells - in contrast to siRNAs packaged into particles designed to facilitate siRNA passage into cells and conceal them from surveillance by Toll-like receptors. But comments that clinical trials should be approached with 'great caution' are overwrought. Additionally, the findings do not reflect how most therapeutic siRNAs are actually designed. Avoiding generalized cytokine responses by direct testing and subsequent chemical modification is now standard. Sequencespecific microRNA-like 'off-target effects' have yet to be shown to limit the therapeutic potential of siRNAs.

Professor, Department of Biochemistry \& Molecular Pharmacology, University of Massachusetts Medical School, Worcester, Massachusetts, USA

COMPETING INTERESTS STATEMENT

The author declares competing financial interests: details accompany the fulltext HTML version at http://www.nature.com/naturemedicine,

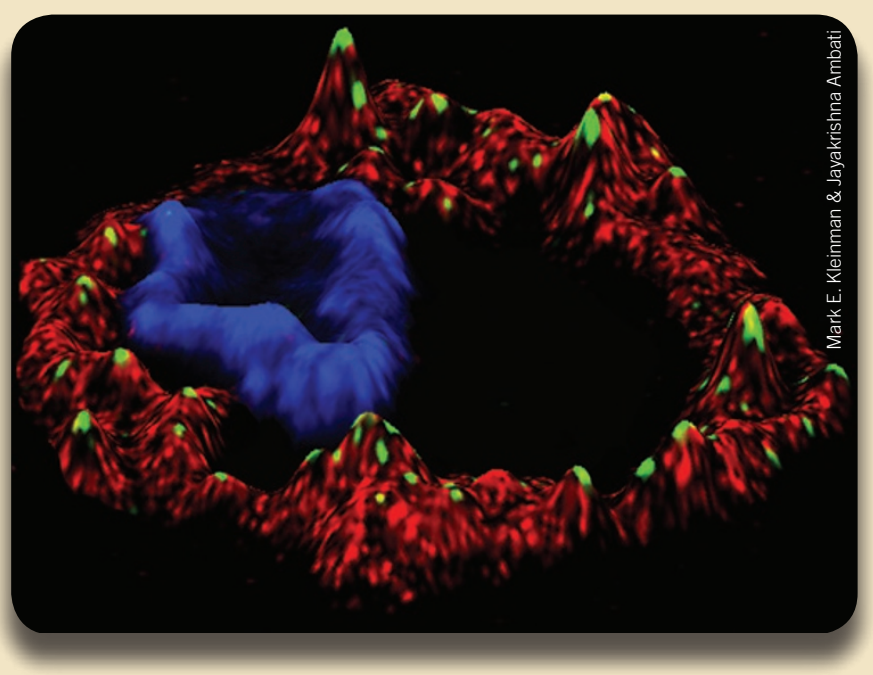

Surface map of a human choroidal endothelial cell exposed to siRNA (red). siRNA does not enter the cell but instead 'paints' the surface, which is studded with toll-like-receptor 3 (green). DNA in blue.

\section{Mark A. Kay:}

As this study suggests, the preclinical efficacy observed for this technique in mouse models of macular degeneration probably resulted from nonspecific side effects rather than RNAi-mediated gene targeting - suggesting that these clinical trials will not be the anticipated proof-of-concept for the platform in humans. Moreover, it is likely that nonspecific effects plague other reported siRNA-knockdown studies.

On a positive note, the range of nonspecific effects observed in siRNA studies is becoming better defined, as well as approaches to reduce them. Moving forward, more rigorous experimental standards will be needed to establish specific gene knockdown in the absence of nonspecific responses in the best available animal models of human disease.

Professor, Departments of Pediatrics and Genetics, Stanford University, Stanford, California, USA.

${ }^{1}$ Kleinman, M.E., Yamada, Y. et al. Sequence- and target-independent angiogenesis suppression by siRNA via TLR3. Nature 452, 591-597 (2008). 
E R R A TA

\section{Erratum: Wandering eye for RNAi}

John Rossi

Nat. Med. 14, 611 (2008); published online 5 June 2008; corrected after print 6 June 2008

In the version of this article initially published, the competing financial interests statement was missing for this author. The author declares competing financial interests: details accompany the full-text version of the paper at http://www.nature.com/naturemedicine/. 